\title{
Research of Drainage Gas Recovery Technology in Gas Wells
}

\author{
Shuren Yang1, Di Xu' ${ }^{1}$ Lili Liư1, Chao Duan², Liqun Xiu ${ }^{1}$ \\ ${ }^{1}$ Department of Petroleum Engineering, Northeast Petroleum University, Daqing, China \\ ${ }^{2}$ CNOOC (China) Limited Zhanjiang Branch, Zhanjiang, China \\ Email: wonderdi@sina.com
}

Received 14 April 2014; revised 14 May 2014; accepted 21 May 2014

Copyright (C) 2014 by authors and Scientific Research Publishing Inc.

This work is licensed under the Creative Commons Attribution International License (CC BY).

http://creativecommons.org/licenses/by/4.0/

(c) (i) Open Access

\begin{abstract}
Drainage gas recovery technology is the main method of gas recovery process in gas field, which has domestically and internationally been the main gas recovery processing measure in later stage of gas field production. In this context, produced water or condensate liquid will not be carried out of pit shaft by natural gas with the gradual drop of gas reservoir pressure and natural gas flow velocity, thus they will remain in pit shaft and form the so-called "gas well gathered liquid". This fluid severely affects natural gas output and leads to the decline of oil field economic benefits, thus drainage gas recovery measure must be taken to increase gas well or even gas field output. It becomes the primary problem to be solved to select the best drainage gas recovery measure which can maximize gas field benefits and optimize gas well safety.
\end{abstract}

\section{Keywords}

Drainage Gas Recovery, Process Optimization, Critical Liquid-Carrying Flow Rate, Technical Index

\section{Introduction}

The purpose of this research is to establish a set of gas drainage way to determine the optimum process technical solution methods and procedures, according to various special geological condition and the gas wells in the production status.

Building such a comprehensive software platform will make the methods and techniques of drainage gas recovery have a new progress, make the management work well established in the scientific basis, so as to reduce the unreasonable factors, improve the quality of the design of the gas well process, and improve the overall economic benefit of gas field. So, this topic research has a very important and realistic significance. The practice experience shows that "draining gas extraction technology" plays a large role for the gas well steady-yield, and 
improves the recovery. So how to choose dewatering gas technology of more in line with the gas well, stronger adaptability and larger displacement and good economic performance, has become an issue that is worth studying. The related literature and achievement is not much, so research on this topic has a very vital significance [1].

\section{Basic Mathematic Model and Design Procedure of Optimized Column Drainage Gas Recovery Process}

From optimized tube drainage gas recovery theory we can know that critical flow rate, critical flow velocity, correlation flow rate and correlation flow velocity of gas well continuous drainage can be determined by formulas below [2]:

$$
\begin{gathered}
q_{k p}=0.648\left(\gamma_{g} Z T\right)^{-\frac{1}{2}}\left(10553-34158 \frac{\gamma_{g} p_{w f}}{Z T}\right)^{\frac{1}{4}} p_{w f}^{\frac{1}{2}} d_{i}^{2} \\
u_{k p}=0.03313\left(10553-34158 \frac{\gamma_{g} p_{w f}}{Z T}\right)^{\frac{1}{4}}\left(\frac{\gamma_{g} p_{w f}}{Z T}\right)^{-\frac{1}{2}} \\
u_{r}=\frac{u}{u_{k p}} \\
q_{r}=\frac{q_{s c}}{q_{k p}}
\end{gathered}
$$

When actual parameters of gas wells can not reach critical flow parameters, rational oil tube diameter should be re-selected to ensure continuous drainage by formula below:

$$
d_{i}=1.2433\left(\gamma_{g} Z T\right)^{\frac{1}{4}}\left(10553-34158 \frac{\gamma_{g} p_{w f}}{Z T}\right)^{-\frac{1}{8}} \times p_{w f}^{-\frac{1}{4}} q_{s c}^{\frac{1}{2}}
$$

where: $q_{s c}$ gas volume flow rate under standard state, $10^{3} \mathrm{~m}^{3} / \mathrm{d}$;

$q_{k p} \longrightarrow$ critical flow rate established when gas well continuous drainage under standard state, $10^{3} \mathrm{~m}^{3} / \mathrm{d}$;

$q_{r}$-dimensionless correlation flow rate of gas well;

$u_{k p}$ as volume flow rate of tubing shoe fracture at gas well bottom, $10^{3} \mathrm{~m}^{3} / \mathrm{d}$;

$u \_$gas flow velocity under standard state of gas well, $\mathrm{m} / \mathrm{s}$;

$u_{r}$ dimensionless correlation flow velocity of gas flow at tubing shoe;

$P_{w f}$ abstract well bottom pressure at tubing shoe, $\mathrm{MPa}$;

$T, Z$ _

$\gamma_{g} \_$natural gas relative density;

$d_{i}$ —- design oil tube inner diameter, $\mathrm{cm}$.

We can design the continuous optimized tube Nomograph of outlet wells by from Formula (2-1) to formula (2-5). Applied design applied Formula (2-1) to Formula (2-5) and Nomograph is summarized below:

1) Based on flowing tube diameter $d_{i}$, well depth $H_{i}$, output $q_{s c}$, well bottom flowing pressure $P_{w f}$, natural gas relative density $\gamma_{g}$ and other parameters. We can get gas well continuous drainage flow rate $q_{k p}$ and correlation parameter $q_{r}$ by Formula (2-1) and Nomograph, thus we can judge gas well working system and drainage capacity;

2) When $q_{r} \geq 1$ and gas well can lift liquid continuously, and gas well can achieve relative stable "tri-stable" working system of pressure, production and gas/water ratio. When $q_{r}<1$, gas well can not lift liquid continuously, we could re-optimize tube diameter $d_{i}$ by Formula (2-2) and Nomograph and repeat procedure (1) to ensure $q_{r} \geq 1$, thus regular production could go on with new flowing tube diameter $d_{i}$;

3) From the possible established maximum pressure drop $\left(\Delta P=P_{w f}-P_{w h}\right)$ of gas well, checkout whether inlet pressure is higher than transiting pressure at the flowing tube diameter we got to ensure that consumers or collecting lines can get natural gas. If well head pressure is larger than transiting pressure, then the calculated results can be adopted, or gas well will select lager diameter by Formula (2-5); 
For large water production gas wells, when it can not work regularly even large diameter oil tubes are applied, we can work out $q_{r}$ by gas well equivalent oil tube diameter. When $q_{r} \geq 1$, and there is no danger to erode casing tubes, casing tubes can be used in production. With technical clues above, work out a computer program to design continuous drainage column [3].

\section{The Model Establishment for Critical Flow Carrying Liquid in Gas Wells}

In 1969, Turner compares the model of the constant moving liquid films in the walls and the model of gas current of high velocity carrying liquid. The liquid films model describes the process for films to climb up to wall, but the calculation is difficult. The model of gas current of high velocity carrying liquid describes the drops in the center of the high-velocity gas current. Both the model of the liquid films and the model of gas current of high velocity carrying liquid exist in the actual production. Besides, the films in the walls will exchange medium with the drops in the gas current. The films descend and break into drops. Plenty of researches indicate that the model of gas current of high velocity carrying liquid is better for the problem of liquid loading in the gas wells. Turner presumed the drops in the wells as balls and deduced the calculations of the minimum gas flow rate and the minimum production if the gas can carry the liquid in the gas wells. Later Professor Li Min in Southwest Petroleum University proposed the ellipsoid model while Engineer in Liaoning Oil Exploration Bureau the cone model.

The chapter discusses Turner’s ball model, Professor Li Min’s ellipsoid model and Engineer Zhongyi Wang's cone model and provides the formulas of forecasting the minimum flow rate and critical production.

\subsection{The Force Analysis of Drops}

The drops in the gas wells are under the stress of the buoyancy from ambient gas $F_{g}$, their own gravity $G_{w}$ and the drag force of gas $F_{D}$. Where:

$$
\begin{gathered}
G_{w}=\rho_{l} V g \\
F_{g}=\rho_{g} V g \\
F_{D}=C_{D} S \Delta p \\
\Delta p=\rho_{g} v_{g}^{2} / 2
\end{gathered}
$$

where: $V$ - the volume of the liquid column, $\mathrm{m}^{3}$;

$S$ - the drops' projected area in the direction of movement, $\mathrm{m}^{2}$;

$C_{D} \_$drag coefficient;

$\triangle P$ — the flowing pressure impacting the drops, $\mathrm{Pa}$;

$V_{g}$ — the velocity of gas current, $\mathrm{m} / \mathrm{s}$.

If the drops in the gas wells can be carried out of the wellbore, the sum of the buoyancy and the drag force is more than the gravity of drops, namely

$$
F_{g}+F_{D} \cos \theta>G_{w}
$$

The following formula can be obtained after calculating:

$$
u_{c r}>\sqrt{\frac{2\left(\rho_{l}-\rho_{g}\right) V g}{C_{D} S \rho_{g} \cos \theta}}
$$

where: $\theta$-hole deviation angle;

$u_{c r}$ critical flow rate carrying liquid in gas wells, $\mathrm{m} / \mathrm{s}$.

The critical flow rate carrying liquid in gas wells $u_{c r}$ can be described as:

$$
u_{c r}=\sqrt{\frac{2\left(\rho_{l}-\rho_{g}\right) V g}{C_{D} S \rho_{g} \cos \theta}}
$$

In the Formula (3-7), $\rho_{l}, \rho_{\mathrm{g}}, \theta$ can be obtained in the regular production. So the critical flow rate carrying liquid is mainly decided by the shape of the drops and the drag coefficient. 


\subsection{The Shape of Drops}

1) The ball model

Turner deduced the critical flow rate carrying liquid in the high gas-water ratio and flow in 1965. He assumed the drops in the gas wells as balls.

Calculate the $V$ and $S$ of the ball in the Formula (3-7).

$$
V=\frac{\pi d_{d}^{3}}{6}
$$

where: $d_{d}$ — the diameter of the drops in the shape of ball, $\mathrm{m}$.

$$
S=\frac{\pi d_{d}^{2}}{4}
$$

Substitute (3-8), (3-9) into (3-7), the following formula can be reached:

$$
u_{c r}=\sqrt{\frac{4\left(\rho_{l}-\rho_{g}\right) d_{d} g}{3 C_{D} \rho_{g}}}
$$

It can be seen in the Formula (3-10) that the flow rate needed for gas to carry drops is proportional to the diameter of drops. If the gas current can carry the biggest drop to the earth, then the liquid in the hole won't get together in the bottom of the well. How to determine the minimum diameter of the drops can be solved by Weber number. When the drops are carried upwards, they suffer two kinds of force. One is velocity pressure to crush the droplet, namely the inertia force, the other one is surface tension to keep it complete. Weber number is the ratio of these two forces.

$$
N_{w e}=\frac{v_{g}^{2} \rho_{g}}{\sigma / d_{d}}=\frac{v_{g}^{2} \rho_{g} d_{d}}{\sigma}
$$

where: $\sigma$ — the liquid surface tension, $\mathrm{N} / \mathrm{m}$.

After many experimental analyses, the researchers determined the critical value to maintain the drops as 30, namely the critical value of Weber number. It can be seen in the Formula (3-11) that the square of the gas flow rate is proportional to Weber number. When the gas flow rate is large enough to reach the critical Weber number, the drops will break under the inertia force. Substitute Weber number $=30$ into (3-11) and work out the diameter $d_{d}$, which is the maximum diameter to maintain the drops steady.

$$
d_{\max }=\frac{30 \sigma}{\rho_{g} v_{g}^{2}}
$$

where: $d_{\max }$ the maximum diameter of the ball drops, $\mathrm{m}$.

Substitute (3-12) into (3-10), and deduce the minimum gas flow rate carrying the biggest drops.

$$
u_{c r}=\left[\frac{4 g \sigma\left(\rho_{l}-\rho_{g}\right)}{C_{D} \rho_{g}^{2}}\right]^{0.25}
$$

2) The ellipsoid model

$\mathrm{Li}$ Min considers that when the drops move in the high velocity gas current, there are differential pressures before and after them. Liquid is flat under the pressure. The flat drop maintains under the surface tension and differential pressure. The equilibrium condition is as follows (The work acted on the drops for differential pressure is equal to the surface work as the surface tension of drops changes):

$$
\Delta p S \mathrm{~d} h_{d}+\sigma \mathrm{d} S=0
$$

where: $h_{d}$ the height of the flat drops, $\mathrm{m}$.

As the drops become flat from ball and the volume is unchangeable, then

$$
V=S h_{d}
$$


According to the Formula (3-14), the following formula can be obtained:

$$
\Delta p S / \sigma=-\mathrm{d} S / \mathrm{d} h_{d}
$$

The following formula can be obtained from (3-15):

$$
S=V / h_{d}
$$

Derivate the two sides of (3-17) for $h_{d}$ :

$$
\mathrm{d} S / \mathrm{d} h_{d}=-V / h_{d}^{2}=-S / h_{d}
$$

From (3-16) and (3-18), the following formula can be reached:

$$
h_{d}=2 \sigma /\left(\rho_{g} u_{g}^{2}\right)
$$

Substitute $V / S=h_{d}=2 \sigma /\left(\rho_{g} u_{g}^{2}\right)$ into (3-7), the following formula can be obtained:

$$
u_{c r}=\left[\frac{4 g \sigma\left(\rho_{l}-\rho_{g}\right)}{C_{D} \rho_{g}^{2}}\right]^{0.25}
$$

3) The cone model

Zhongyi Wang model considers the deformation of drops movement, which is the same as Li Min model. However, Zhongyi Wang considers the drops as cones and calculates.

The following formula can be obtained after calculating:

$$
V / S=2 \sigma /\left(3 \rho_{g} u_{g}^{2}\right)
$$

Substitute into (3-7):

$$
u_{c r}=\left[\frac{4 g \sigma\left(\rho_{l}-\rho_{g}\right)}{3 C_{D} \rho_{g}^{2}}\right]^{0.25}
$$

\subsection{Theory Comparison of Three Continuous Liquid Carrying Models}

Based on assumed conditions before and liquid force balance model, Professor Zhongyi Wang and Ming Li respectively build ball cap model and ellipsoid model. They actually are the selection of drag force factor Cp, which is mainly affected by turbulent flow, gas phase, compressibility and non-spherical grain. Comparison of the three models is shown in Table 1.

We can see from Table 1, the improvements of three models focus on the shape of liquid. About drag force factor, sphere model has the smallest drag force factor, while ball cap model has the largest. We can know that drag force factor increases dramatically with the raise of effective flow-facing areas.

\subsection{Calculation of Critical Liquid Carrying Flow Rate}

When daily output of a gas well is lower than critical liquid carrying flow rate, the gas well will gather liquid; the calculating results and practical situations comparison of three wells in three models are shown in Table 2.

From Table 2 we can know that Turner model is most suitable for practical situations. The maximum critical liquid carrying flow rates and gathering-liquid judgments got from Ming Li model and Zhongyi Wang model have some deviation.

Critical liquid carrying flow velocity formula:

$$
V_{c r}=5.5\left[\frac{\sigma\left(\rho_{1}-\rho_{2}\right)}{\rho_{g}^{2}}\right]^{0.25}
$$

Corresponding critical liquid carrying flow rate formula:

$$
q_{c r}=2.5 \times 10^{4} \frac{A p V_{g}}{Z T}
$$


Table 1. Continuous liquid carrying models comparison.

\begin{tabular}{cccc}
\hline Model & Liquid shape & Drag force factor & Critical liquid carrying velocity \\
\hline Turner model & Sphere & 0.44 & $V_{c r}=5.5\left[\frac{\sigma\left(\rho_{1}-\rho_{2}\right)}{\rho_{g}^{2}}\right]^{0.25}$ \\
Ming Li model & Ellipse & 1 & $V_{\text {cr }}=2.5\left[\frac{\sigma\left(\rho_{1}-\rho_{2}\right)}{\rho_{g}^{2}}\right]^{0.25}$ \\
Zhongyi Wang model & Ball cap model & 1.17 & $V_{\text {cr }}=1.8\left[\frac{\sigma\left(\rho_{1}-\rho_{2}\right)}{\rho_{g}^{2}}\right]^{0.25}$ \\
\hline
\end{tabular}

Table 2. Comparison of calculating results and practical situations.

\begin{tabular}{|c|c|c|c|c|c|c|c|c|}
\hline \multirow{3}{*}{$\begin{array}{c}\text { Number } \\
1\end{array}$} & \multirow{3}{*}{$\begin{array}{c}\text { Daily output }\left(10^{4} \mathrm{~m}^{3} / \mathrm{d}\right) \\
4.51332\end{array}$} & \multicolumn{6}{|c|}{ Critical liquid carrying flow rate $\left(10^{4} \mathrm{~m}^{3} / \mathrm{d}\right)$} & \multirow{3}{*}{$\begin{array}{c}\text { Practical results } \\
\text { No liquid }\end{array}$} \\
\hline & & \multicolumn{2}{|c|}{ Zhongyi Wang model } & \multicolumn{2}{|c|}{ Ming Li model } & \multicolumn{2}{|c|}{ Turner model } & \\
\hline & & 1.4638 & No liquid & 2.0334 & No liquid & 4.4733 & No liquid & \\
\hline 2 & 1.0445 & 1.4664 & Liquid & 2.0365 & Liquid & 1.4805 & Liquid & Liquid \\
\hline 3 & 5.0904 & 1.95 & No liquid & 2.7362 & No liquid & 6.0196 & Liquid & Liquid \\
\hline
\end{tabular}

where: $q_{c r}$ the minimum flow rate or unloading flow rate required for gas carrying liquid, $10^{4} \mathrm{~m}^{3} / \mathrm{d}$;

$A-$ section area of oil pipes, $\mathrm{m}^{2}$;

$p$-bottom hole flowing pressure, MPa;

$\mathrm{T}$ —bottom hole gas temperature, $\mathrm{K}$;

Z__ gas compressibility factor under specific bottom hole flowing pressure and gas temperature.

Formula (3-10) and (3-11) are minimum gas flow velocity and minimum gas flow rate practical formulas derived from thoughts of Turner and others. They are suitable for gas-water wells and gas-condensate oil wells.

Because of lacking physical property data of surface tension under different pressure and temperature, approximate calculation could adopt values below:

For water: $\sigma=60 \times 10^{-3} \mathrm{~N} / \mathrm{m}, \rho_{\mathrm{l}}=1074 \mathrm{~kg} / \mathrm{m}^{3}$;

For condensate oil: $\sigma=20 \times 10^{-3} \mathrm{~N} / \mathrm{m}, \rho_{\mathrm{l}}=721 \mathrm{~kg} / \mathrm{m}^{3}$.

As the surface tension and density difference between gas and water are higher than that between oil and gas, physical values of well water mixed liquid are calculated by water for well which products both water and condensate oil.

For better applied in field, two simplified formulas are derived by Turner and others under several assumed conditions, the preconditions of simplified formulas are [4]:

1) $\gamma_{g}=0.6, T=120^{\circ} \mathrm{F}, \rho_{g}=0.0031 P$

2) For water: $\sigma=60 \mathrm{dyn} / \mathrm{cm}, \rho_{L}=67 \mathrm{lb} / \mathrm{ft}^{3}$

3) For condensate oil: $\sigma=20 \mathrm{dyn} / \mathrm{cm}, \rho_{L}=47 \mathrm{lb} / \mathrm{ft}^{3}$

Then, for water:

$$
v_{g}=\frac{5.62 \times(67-0.0031 p)^{0.25}}{(0.0031 p)^{0.5}}
$$

For condensate oil:

$$
v_{g}=\frac{4.02 \times(45-0.0031 p)^{0.25}}{(0.0031 p)^{0.5}}
$$

Minimum flow rate formula is: 


$$
q_{s c}=3.06 \frac{A p v_{g}}{Z T}
$$

where: $q_{s c}$ the minimum flow rate or unloading flow rate required for gas carrying liquid, $10^{6} \mathrm{~m}^{3} / \mathrm{d}$;

$A-$ section area of oil pipes, $\mathrm{ft}^{2}$;

$p \_$bottom hole flowing pressure, psi;

$T$ — bottom hole gas temperature, ${ }^{\circ} \mathrm{F}$;

$Z$ _ _ gas compressibility factor under specific bottom hole flowing pressure and gas temperature;

$v_{g} \_$minimum unloading flow rate, $\mathrm{ft} / \mathrm{s}$.

\section{Optimization Software of Drainage Gas Recovery}

This software applicants Visual Basic 6.0 program, convenient operation and clear operator interface, somewhat similar to the Windows operating interface. The main interface is shown in Figure 1. The main menu of the software including "Introduce of Drainage Gas Recovery", "Computational Analysis", "Help” and "Exit” four options, in which "Computational Analysis" is used for calculations mentioned in the previous chapters (including Down-hole Pressure, Critical Flow, Liquid Loading and Column Optimizing). And the "Help" gives various considerations and other explanatory to software installation and operation. The "Exit" is used to end the software, and then return to Windows operating system [5].

Click "Computational Analysis" in the menu interface will appear an interface as follows.

Introduce each sub-menu's functions as follow:

1) Pressure-drop Calculation of Water-producing Gas Well

This Program can calculate the bottom-hole pressure of the gas well using the input parameters. Show in Figure 2.

2) Calculation of Critical Delivery

This Program can calculate the critical delivery and critical velocity of the gas well using the input parameters. Show in Figure 3.

3) Calculation of Liquid Loading

This Program can calculate the liquid loading of the gas well using the input parameters. Show in Figure 4.

4) Column Optimizing

This Program can calculate the liquid loading of the gas well using the input parameters. Show in Figure 5.

\section{Conclusions}

1) According to the actual situation of gas field, the paper analyzes the wellhead back pressure effect on the critical liquid carrying capacity, and Calculation formula of Critical carrying fluid flow velocity and critical fluid flow is derived;

2) Using Turner model to establish critical liquid carrying flow velocity calculation formula of gas well combining field situations based on the character of gas well liquid carrying. We can also establish critical liquid

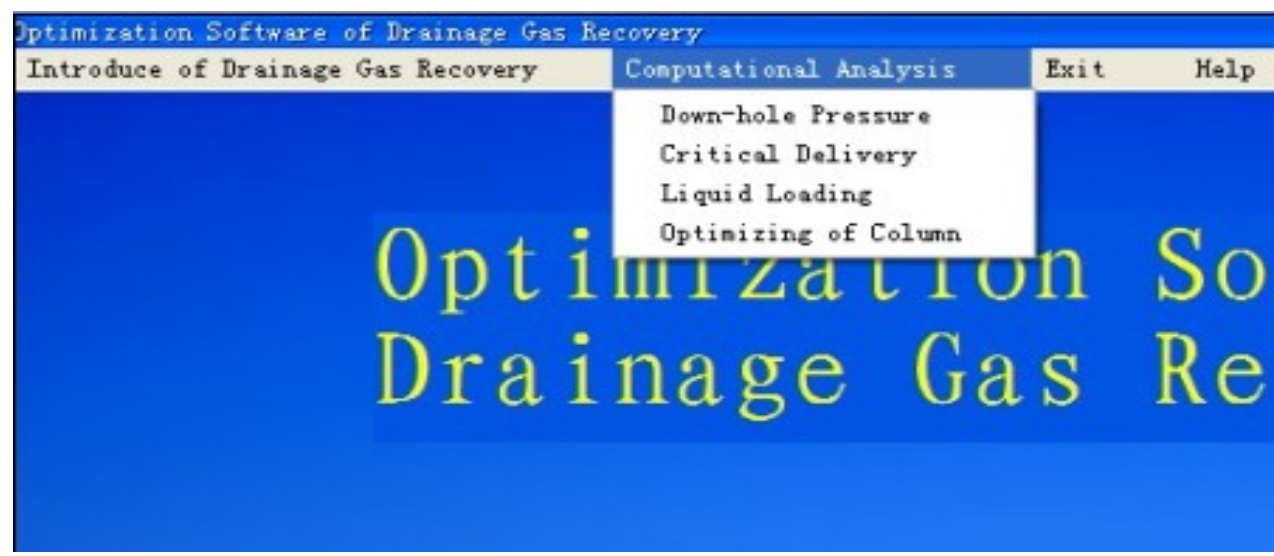

Figure 1. Calculation analysis. 


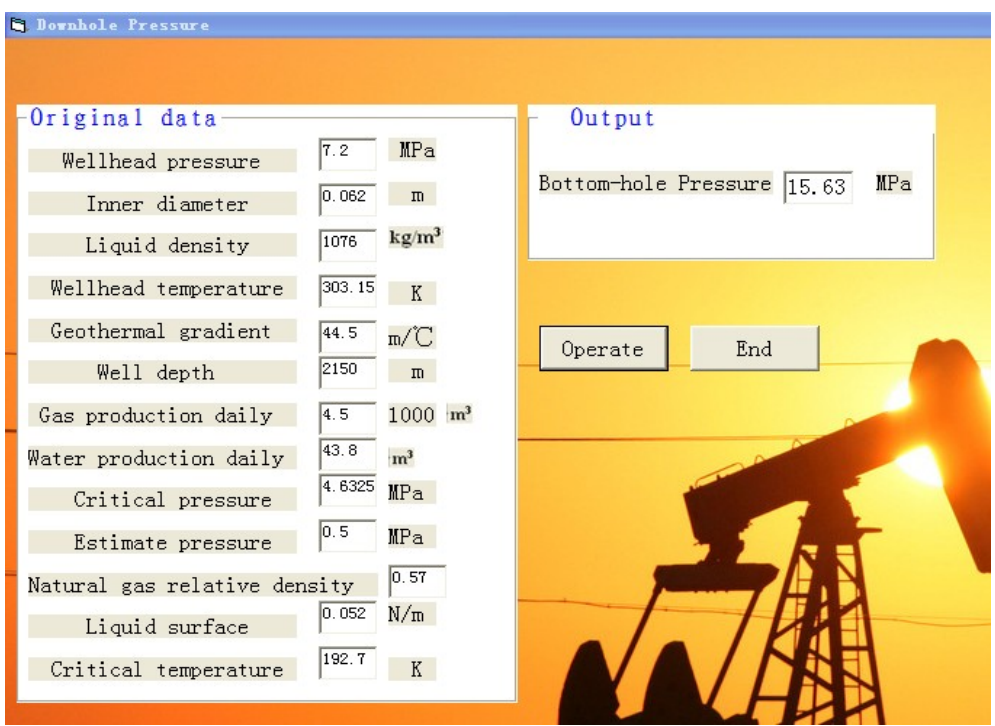

Figure 2. Interface of down-hole pressure calculation.

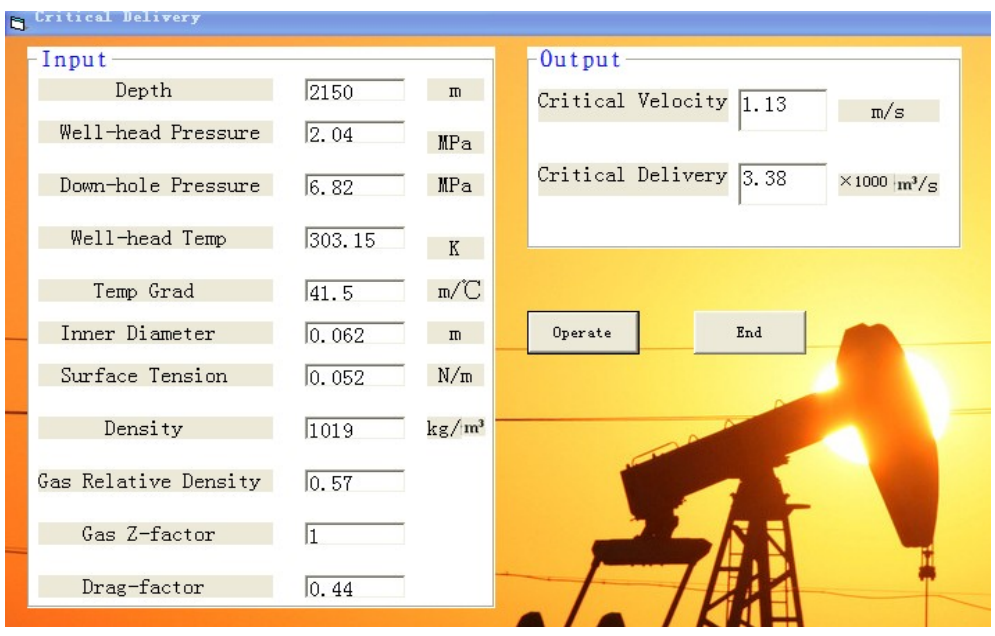

Figure 3. Interface of critical delivery calculation.

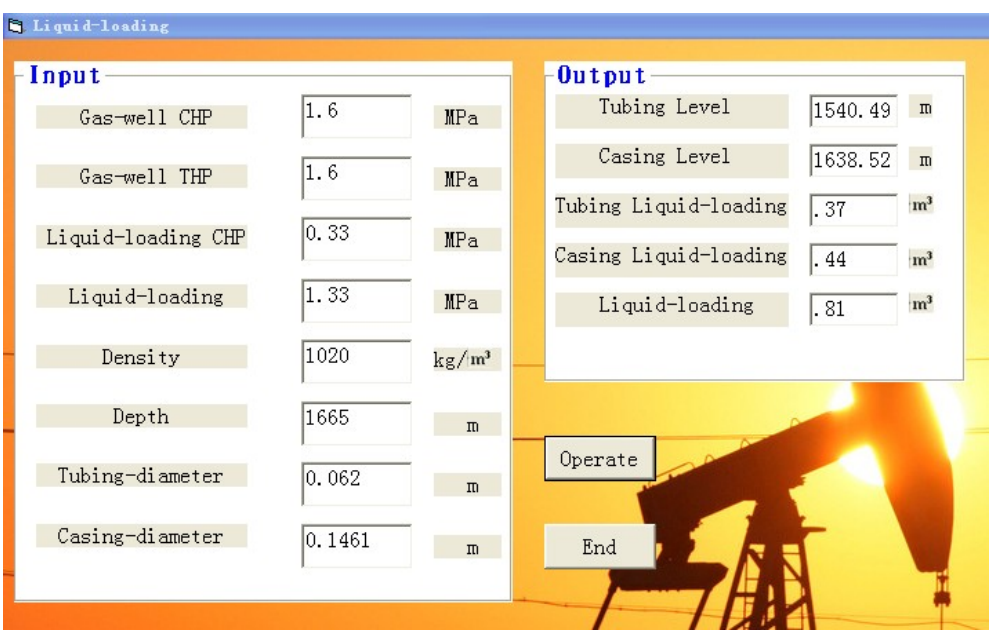

Figure 4. Interface of liquid loading calculation. 


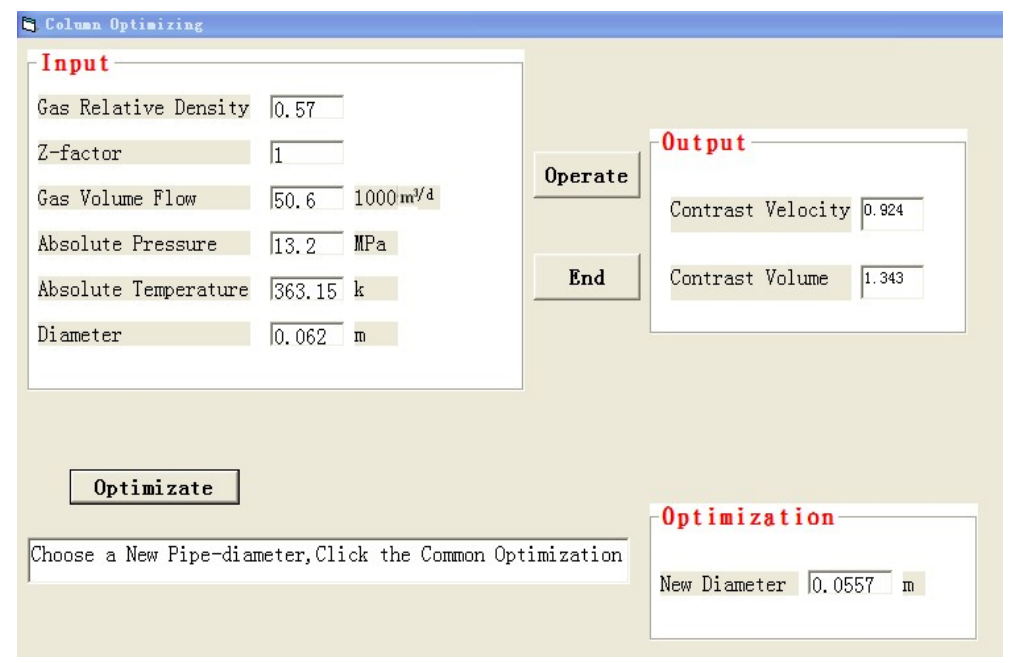

Figure 5. Interface of column optimizing.

carrying flow rate calculation formula of gas well referring to critical liquid carrying flow velocity calculation formula of gas well. Practical application shows that established formulas could meet requirements of gas well liquid gathering judgment;

3) According to the models, the optimization designing optimized software of dewatering gas recovery suits for the majority of oil and gas field.

\section{References}

[1] Lage, A.C.V.M. and Time, R.W. Mechanistic Model for Upward Two-Phase Flow in Annulus. SPE 63127.

[2] Li, Z.Y., Ma, J.Z. and Liao, Z.C. (2008) Research of Drainage Gas Recovery Technology. Inner Mongolia Petrochemical Industry, 11.

[3] Zhou, M.Q., Duan, Y.-G., et al. (2010) Research and Application on Drainage Gas Recovery by Gas Well Self-Energy. Journal of Chongqing Technology Institute (Natural Science Edition), 10.

[4] Wu, Y.T. (2009) The Preference of Drainage Gas Recovery Methods and Safety Assessment of Gas Well with Water Drainage. China University of Petroleum, 4.

[5] Zheng, X.X. (2008) Drainage Gas Recovery Method Optimization. China University of Petroleum, Beijing. 